\title{
Relevance of a neutral cue in a two-choice detection task in the rat
}

\author{
CARLOS M. HAMAME, PAUL H. DELANO and LUIS ROBLES
}

Programa de Fisiología y Biofísica, Instituto de Ciencias Biomédicas, Facultad de Medicina, Universidad de Chile, Santiago, Chile

\begin{abstract}
Relevance of a neutral cue for performance in a two-choice visuospatial detection task was examined. Nine rats were trained, 5 with short intertrial interval (ITI) and 4 with long ITI, to detect a target (lateral lights) presented after a neutral-cue (central light). The removal of the neutral-cue decreased accuracy and increased response latencies and omissions. These results demonstrate that a neutral-cue, preceding the target, is relevant for the performance, suggesting that rats are highly expectant during the neutral-cue and reallocate attentional resources during ITI. Furthermore, latencies were higher, omissions were lower and the fall of accuracy was greater for rats with long than with short ITI, which could indicate that the neutral-cue was more relevant for the former group.
\end{abstract}

Key terms: attention, expectancy, neutral cue, attentional task, rat.

\section{INTRODUCTION}

It generally is recognized that attention is not a simple construct, but that it comprises several processes: a) vigilance or sustained attention, a state of continuous readiness to respond to unpredictable events; b) selective attention in which the subject has to focus on one perceptual modality or on one dimension of a stimulus, while ignoring the rest; c) divided attention or parallel processing in which the subject allocates processing resources between the execution of two or more tasks (Bushnell, 1998a; Muir, 1996); d) orienting, which has been used in several paradigms, such as observation of unconditioned behavior and cued target detection (including here the covert orienting paradigm); e) expectancy, as a readiness of the nervous system to receive particular stimuli and to generate particular responses (Bushnell, 1998a; Pang et al., 1993).

In order to assess such a complex construct, and its relationship with the functioning of the nervous system, several studies have searched for ways to relate rodents' performance in behavioral tasks with attentional processes, attempting to develop rodent models of human attentional functions (see Robbins, 2000; Steckler and Muir, 1996; Bushnell, 1998a; Muir, 1996; D'Mello and Steckler, 1996, for reviews on the subject).

Visual two-choice reaction time tasks have been used to assess a variety of attentional processes and their relationship with several physiological and pharmacological variables. In sustained attention studies (Grilly and Gowans, 1988; Grilly et al., 1998; Sahgal, 1988), the critical parameter is the predictability of the target, which is related to the duration and variability of the intertrial interval (ITI), among other things.

Stoher et al. (1997) developed a visual two-choice task in order to model attentional shift and thereby isolate whether it was aging or the loss of cholinergic basal forebrain neurons that produces the difficulties of Alzheimer patients to shift visuospatial attention. In their task, each 
trial began with both levers retracted. The lighting of a house light was followed by illumination of a central light for two seconds, predicting the illumination of one of the two lateral lights $(2,0.8$ or $0.08 \mathrm{~s})$. The levers then were presented for pressing, avoiding premature responses. There was no ITI period, but the rat had $10 \mathrm{~s}$ to respond. If the response was correct, the next trial began immediately, if incorrect the animal had a two seconds time-out in darkness before the next trial began. The signaling of each trial by a central light made the target a predictable event, which ruled out the sustained attention component of other two-choice tasks (Grilly and Gowans, 1988; Grilly et al., 1998; Sahgal, 1988) and centered the measures on the attentional shift between the two targets.

Another rodent model of attention that involves a two-choice task is Bushnell's (1995) overt orienting paradigm. This is an attempt to model covert orienting in humans, which is the shift of attention from a visual fixation point without moving the eyes (Posner, 1980). In this behavioral procedure, the rat initiates the trial by nose poking in a central food cup, followed by a visual cue onset that signals the location in which the target is going to appear (i.e., left or right of the operant chamber). In this task, Bushnell tested different conditions, such as: no cue; different timing between cue and target onset; invalid cues; and neutral cue (onset of the two cues). Although results were similar to those found in human literature, Bushnell (1995; 1998a) concluded that it is not possible to be sure about the rats visual fixation during the cue presentation. Therefore, the attentional shift could be produced with a motor-orienting response (overt orienting with eyes only or even with the head). Data showed that valid cues (signals target correct location) improved performance of the rats, while invalid cues decreased accuracy and increased reaction time. In the case of neutral cues, the study found little effect on accuracy and time reaction compared to the no cue condition.

We propose that a neutral cue delimits high and low expectancy time periods by signaling the time of occurrence of the discrimination task. The neutral cue allows the animal to attend the front panel, where the stimuli will appear, only when necessary, and permits reallocation of attentional resources during the ITI. In contrast, the absence of the neutral cue makes the task more like a sustained attention task due to the difficulty in predicting the occurrence of the target. It is possible that the neutral cue could be more relevant for performance when the ITI is longer, because the presentation of the target is more unpredictable.

The purpose of this study was to test the relevance of a neutral cue, in delimiting attentional load for different durations of the ITI in a two-choice detection task. To this end, nine male hooded Lister (LongEvans) rats were trained in the task, five with a short ITI and four with a long ITI, until they reached and maintained criterion (above $80 \%$ of accuracy and below $20 \%$ of omissions) for five consecutive days. After that, the neutral cue was removed, keeping constant all other parameters of the twochoice task. Several behavioral indicators, such as accuracy, mean latency of response, percentage of omissions, and response rates were compared for the two conditions, with and without the neutral cue.

\section{METHOD}

\section{Subjects}

Nine male Long-Evans rats (Animal Facility of the Faculty of Medicine, University of Chile), weighing between 250-350 $\mathrm{g}$ at the start of training, were used. They were individually housed in temperature-controlled conditions with an alternating dark-light cycle (lights off from 8:00 to 20:00). All rats were given water $a d$ libitum and were food deprived, maintaining 80 to $90 \%$ of their free-feeding weight during the experimental period. All animals were trained five days a week, over approximately two months with two sessions of 50 trials each day, except for the testing phase that consisted of one session of 50 trials on ten consecutive days. Animals were divided in two groups, one 
with a short ITI (s_ITI) that varied randomly from 7 to $13 \mathrm{~s}$, and the other with a long ITI (1_ITI) that varied between 27 and $33 \mathrm{~s}$. All procedures involving the animals were made in accordance with the local Committee of Bioethics and the Guiding Principles of the Care and Use of Animals approved by the American Physiological Society.

\section{Apparatus}

The apparatus consisted in an operant cage $(25 \mathrm{~cm} \times 31 \mathrm{~cm} \times 31 \mathrm{~cm})$ made of three plexiglass panels, one aluminum front panel and a metal grid floor. The front panel had three different lights: a central light (neutral cue) above a magazine food dispenser and two lateral lights (targets) above two levers that were located six centimeters above the grid floor. The target lights, central light, and the magazine food dispenser were 10 , 14.5 , and $0.5 \mathrm{~cm}$ from the floor, respectively.

\section{Behavioral procedure}

Nine food-deprived rats were trained to perform a two-choice task that consisted of a visuospatial discrimination. Stimuli presentation and data acquisition were controlled by a custom-made computer program (Labwindows (C) system) and a digital interface with a time resolution of 1 ms. After initial shaping of lever-pressing for food reward (dustless precision pellets, $45 \mathrm{mg}$; Bioserv (C) over two to five days, the animals were required to press the right lever when the right light turned on and the left lever when the left light turned on for food reward. Each trial began (see Figure 1) with the onset of the neutral cue (central light) with a random duration (2-4 s), after which randomly, the right or left light (target) was turned on. Target duration was reduced progressively along sessions from 10 to $0.5 \mathrm{~s}$, always maintaining an accuracy $\geq 80 \%$. The ITI had a random duration from 7 to 13 s for group s_ITI and from 27 to 33 s for group 1_ITI. Once the rats were able to perform the discrimination task with an $80 \%$ accuracy and less than $20 \%$ omissions for a $0.5 \mathrm{~s}$ target duration, the test phase began, in which rats were required to stay five consecutive days above criterion in the task. During this period, some behavioral indicators (specified below) were measured. On the sixth day, the neutral cue was removed, and the same behavioral indicators were assessed over the next five days to be compared with the previous performance.

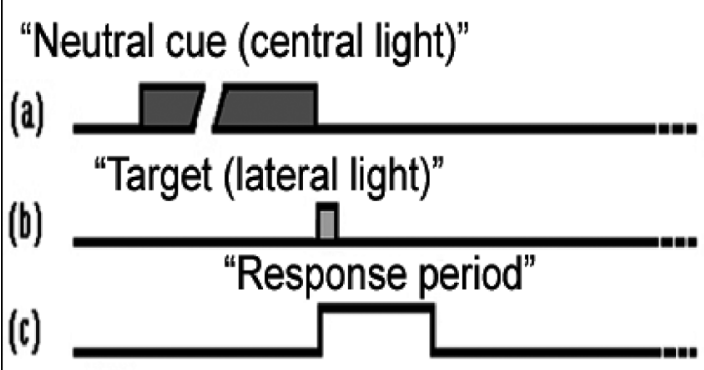

Figure 1: Schematic representation of the temporal sequence of the two-choice detection task. A) Neutral cue (central light) prior to the discrimination (2-4 s); B) Target (lateral light, $0.5 \mathrm{~s})$; C) Response period during and after the presentation of the target $(5 \mathrm{~s})$.

\section{Behavioral variables and data analysis}

The specific behavioral variables measured were: latencies of lever-pressing responses (time between the onset of the stimulus light and the lever-pressing response), accuracy (i.e., \{correct responses / [correct responses + incorrect responses]\} x 100), omission percentage, and number of responses during different periods of the task. The latter were normalized by time, as response rates, during: response period; neutral cue; and ITI (i.e., number of responses / duration of: response period, neutral cue, or ITI). Response latencies, percentage of omissions, accuracy, and response rates were statistically analyzed with a $t$-test for matched pairs, for comparison of the variable means before and after removal of the neutral cue. Latencies, percentage of omissions, response rates, and accuracy (before the removal of the neutral cue) were compared 
between the two groups using a $t$-test for independent samples. Bias indexes were not obtained because the pseudorandom and counter-balanced presentation of the lateral lights prevented bias in correct responses. Statistical analysis was performed using the Statmost32 (C) computer program. The criterion for significance in all tests was $\alpha$ $=.05$.

\section{RESULTS}

Considering all animals from both groups, the average lever-response latency, measured from target onset to lever response, significantly increased after removal of the neutral cue $(t(8)=-4.86, p$ $<.01$, Fig. 2). The mean response latencies of rats from group 1_ITI were larger than those of group s_ITI (by about $140 \mathrm{~ms}$ ) during the five days before $(t(36)=-2.96$, $p<.01)$, but not after removal of the neutral cue due to the variability of the data in the latter stage. The increase in variability after removal of the neutral cue was mainly due to the variability among individuals of the same group (data not shown).

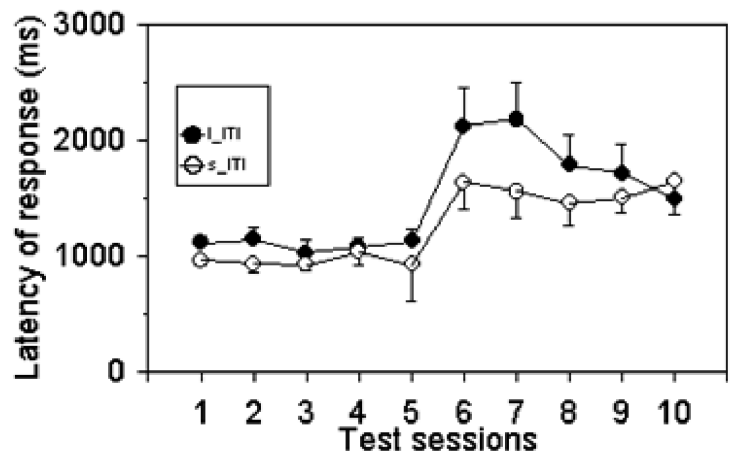

Figure 2: Increase of grand average latencies from group s_ITI (open circles) and group 1_ITI (closed circles) after removal of the neutral cue, $t(8)=-4.86, p<.01$. Mean latency from all animals increased from $1,019 \mathrm{~ms}(S D=142)$ to $1,691 \mathrm{~ms}(S D=448)$. Mean latency, during the five days before removal of the neutral cue, was $956 \mathrm{~ms}(S D=141)$ for group s_ITI and 1,099 ms $(S D=175)$ for group 1_ITI. In this and following figures, error bars depicted.
In both groups, there was an increase in the average percentage of omissions after removal of the neutral cue $(t(8)=-3.6, p<$ .01 , Fig. 3). During the five days with the neutral cue, the mean percentage of omissions from rats of group s_ITI was greater than that of group 1_ITI $(t \overline{7})=3.59$, $p<.01)$. However, as in the case of latencies, the same comparison during the five days after removal of the neutral cue was not significant, mainly due to variability among individuals of the same group.

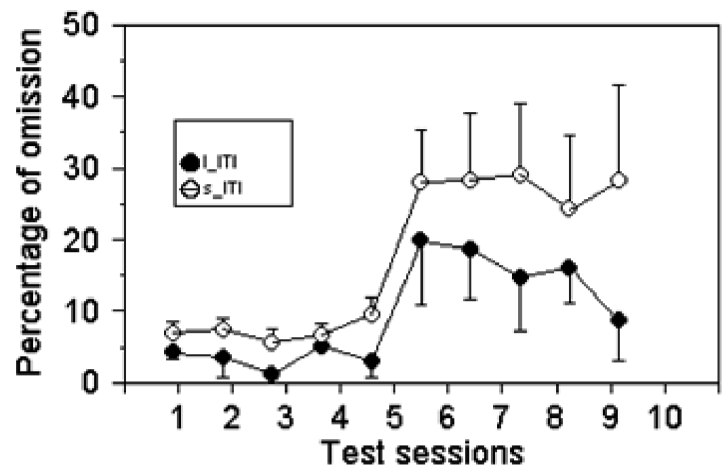

Figure 3: Percentage of omissions before and after removal of the neutral cue in groups S_ITI and 1_ITI. Open circles represent grand average percentage of omissions from group s_ITI and closed circles from group 1_ITI. The percentage of omissions of both groups was 5.9 ( $S D=$ 2.94) before and $20.7(S D=12.78)$ after removal of the neutral cue. Previous to the neutral cue removal, mean percentage of omissions was $7.9(S D=1.85)$ for group s_ITI and $3.4(S D=1.88)$ for group 1_ITI.

All animals had an accuracy above $80 \%$ during the five days previous to the removal of the neutral cue (that percentage was the required criterion to begin the test-phase). Both groups, 1_ITI and s_ITI, fell in their accuracy after removal of the neutral cue $(t$ $(8)=3.57, p<.01$, Fig. 4). However, there was a greater fall in accuracy in group 1_ITI than in group s_ITI during the first two sessions after removal of the neutral cue $(t$ (7) $=3.02, p<.05)$.

No significant correlations were found among accuracy, mean latency, and percentage of omissions, in any of the conditions assessed (Pearson test). 


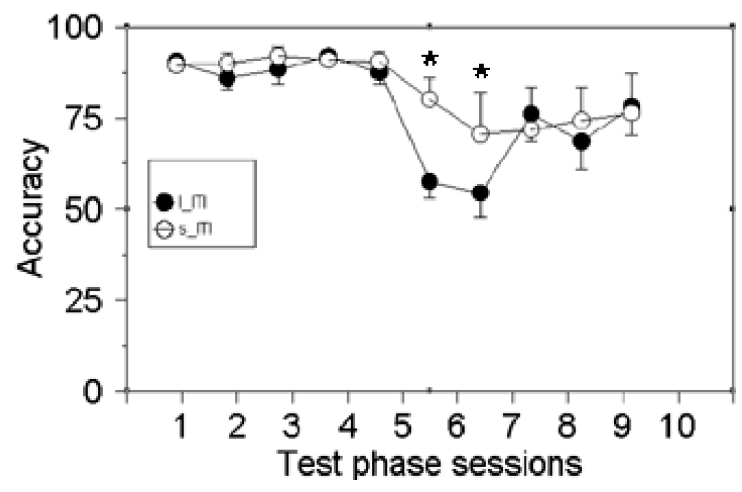

Figure 4: Accuracy before and after removal of the neutral cue in groups s_ITI and 1_ITI. Both groups fell in their accuracy $(t(8)=3.57, p<$ $.01)$ after neutral cue removal. Although, group 1_ITI had a steeper fall than s_ITI $(t(7)=3.02$, $p<.05)$. All animals had a mean accuracy of $89.78(S D=3.46)$ before and $71.11(S D=16)$ after removal of the cue. Asterisks indicate significance of difference between s_ITI and 1_ITI group.

The rate of lever pressing during the response period decreased in both groups, S_ITI $(t(24)=5.09, p<.01)$ and 1_ITI $(t$ $(19)=4.21, p<.01)$, after removal of the neutral cue (Fig. 5A). Furthermore, there was a significant difference in leverpressing rates between the two groups under standard conditions before removal of the neutral cue $(t(7)=-3.7, p<.05)$.

On the other hand, the rate of lever pressing during the ITI significantly increased in both groups, s_ITI $(t(4)=-$ $5.36, p<.01)$ and 1_ITI $(t(3)=-3.39, p<$ .01 ), after removal of the neutral cue (Fig. 5B). A comparison between the ITI leverpressing rates of the two groups before neutral cue removal shows a significantly higher rate in the s_ITI than in the 1_ITI group $(t(14)=2.38, p<.01)$. In addition, the rate of lever pressing during neutral cue presentation was significantly higher in group s_ITI than in group 1_ITI $(t(41)=$ $2.59, p<.01$, Fig. 5C).

Finally, the differences between response rates during the response period and other periods of the task were lower for the s_ITI group than for the 1_ITI group $(t$ (7) $=-3.17, p<.05$ for ITI response rate and $t(3)=-2.83, p<.05$ for neutral cue response rate). This fact indicates a difference in the temporal distribution of responses between the two groups (data not shown).

\section{DISCUSSION}

The behavioral indicators assessed in two different conditions of the two-choice detection task, with and without neutral cue, support the hypothesis that rats were highly expectant during the neutral cue presentation. They also support the notion that this cue facilitated the performance of the rat by delimiting high- and lowexpectancy periods, due to its warning or alerting effect. This issue was explored not only by comparing several behavioral indicators before and after removal of the neutral cue, but also by dividing the rats in two experimental groups under different ITI conditions: one group trained with an ITI of about $10 \mathrm{~s}$ and the other one with an ITI of about $30 \mathrm{~s}$ to assess the different relevance that the cue may have in conditions of variable difficulty of the task. Our data demonstrate that the neutral cue improved rats' performance, as they had shorter response latencies, fewer omissions, and higher accuracy before than after removal of the neutral cue.

Sustained attention has been assessed through two-choice tasks and operant paradigms similar to the one used in this study (Bushnell et al., 1994; Bushnell, 1998b; Givens, 1997; Grilly and Gowans, 1988; Grilly et al., 1998; McCaughy and Sarter, 1995; McGaughy et al., 1996; Sahgal, 1988; Sarter and McCaughy, 1998). A critical factor in the assessment of sustained attention is the unpredictability of the target that has to be detected. This condition is often satisfied in two ways: one, by extending the period of time between presentations of the target; and two, by randomizing this period of time.

In this study, the introduction of a neutral cue signaling the occurrence of the target delimits a period of high attentional load, by turning the detection and discrimination of stimuli during the task into a predictable event in time. This makes 


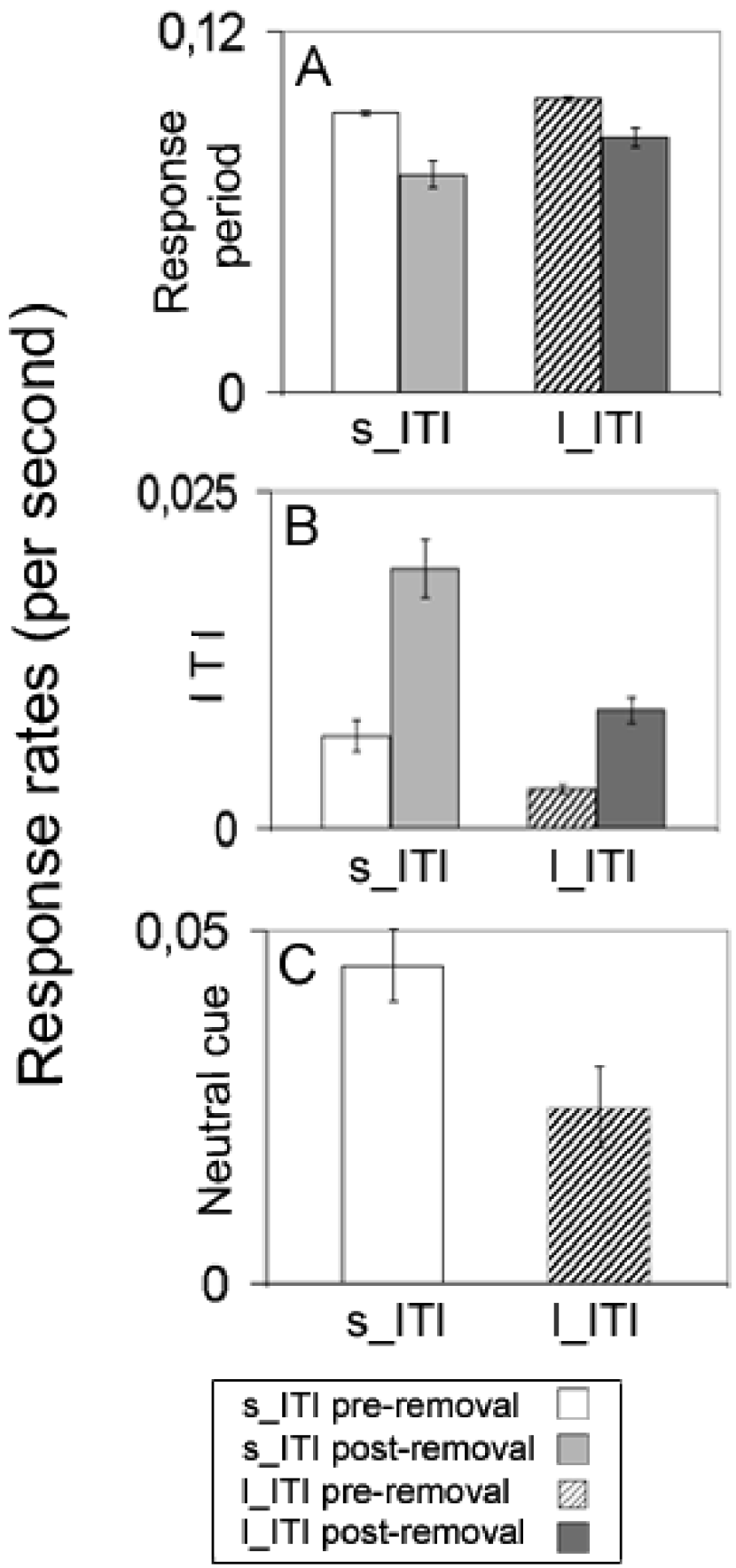

Figure 5: Response rates during different periods of the task. A) Response rates during response period. There was a mean response rate of $0.093(S D=0.004)$ before and $0.072(\mathrm{SD}=0.021)$ after neutral cue removal for group s_ITI and one of $0.098(S D=0.003)$ before and $0.085(S D=0.013)$ after neutral cue removal for group 1_ITI. Both groups together had a mean response rate of 0.093 ( $S D$ $=0.004)$ before and $0.098(S D=0.003)$ after cue removal. B) Response rates during ITI. These rates were $0.007(S D=0.004)$ before and $0.019(S D=0.059)$ after cue removal for group s_ITI. The same rates were $0.003(S D=0.0007)$ before and $0.009(S D=0.004)$ after removal for group 1_ITI. The total mean of ITI lever-pressing rate was $0.013(S D=0.0083)$ for group s_ITI and $0.058(S D=0.0042)$ for group 1_ITI. C) Response rates during presentation of the neutral cue. This rate was $0.044(S D=$ $0.025)$ for group s_ITI and $0.024(S D=0.025)$ for group 1_ITI, before neutral cue removal. 
sustained attention or vigilance during the task unlikely. The existence of two experimental groups with different ITI durations makes it possible to test the relevance of the neutral cue in signaling the occurrence of the target. The cue should be more relevant when the occurrence of the target is more unpredictable, that is, for the group with the longer ITI. It is possible that rats from group s_ITI, due to the short duration of the ITI and hence the lesser unpredictability of the target, could resolve the attentional task making less use of the neutral cue than rats from 1_ITI. This could explain the higher decrease in accuracy that rats from group 1_ITI had during the first two sessions after removal of the neutral cue. Furthermore, it could be argued that the long duration of the ITI for the 1_ITI group led to a better delimitation of expectancy and thus of the temporal distribution of responses. This is indicated by the higher difference of response rates between the response period and other periods of the task in group 1_ITI in comparison with group s_ITI. Responses made during the neutral cue presentation cannot be explained as a generalization, since a previous study using this task (Delano et al., 2003), showed that mean latencies of responses to the target had a peak around $500 \mathrm{~ms}$, while response latencies to the neutral cue were longer than $1,500 \mathrm{~ms}$ and had a flat time distribution.

Mirza and Stolerman (1998), in experiments using a 5-choice serial reaction time task (see Carli et al., 1983, for a description of the task), manipulated several parameters including ITI, and thus event rate, to ascertain aspects of validity for a vigilance task. They showed that, either when augmenting or diminishing event rate, accuracy decreased, latencies increased, and omissions increased. However, it is important to consider that these results were acquired in a single test session, with no observation of posterior stabilization. Changes of indicators in the first session, after manipulation of the event rate, could be transitory unspecific effects of the alteration in task parameters, rather than an effect of the specific manipulation itself.
A cue will shift the attention of the rat to a determinate location where a target is going to appear (Posner, 1980). This is an expected location, and the expectancy of the rat can be influenced by stimulus frequency and repetition (Pang et al., 1992). Responses to frequent stimuli are faster than those to infrequent ones. A perceptual explanation suggests that attention is directed to more frequent stimuli resulting in a better encoding, while a motor explanation emphasizes that animals are more likely to select frequent responses over infrequent ones. Pang et al. arranged different presentation rates in a two-choice discrimination task for modeling human expectancy with rats. The stimulus rates were relative, meaning that if the rate of presentation of the auditory stimulus increased, the rate of the visual stimulus decreased, and vice versa. They demonstrated that, as in human experiments, an increase in stimulus frequency produces a decrease in latency and error rate, and a shift in bias to the more frequent stimulus.

In the present study, the cue is neutral, so it does not carry any information about the specific place in which the target stimulus will appear, however it does signal the timing of presentation of one of the two target stimuli. Group s_ITI had a higher rate of stimulus presentation than group 1_ITI. Consequently, the shorter mean latency of group s_ITI could be explained by the facilitation in stimulus encoding due to the higher frequency of presentation of the targets. This effect has been described before in expectancy tasks, together with lower error rates and better discriminability (Pang et al., 1992; Pang et al., 1993), however in our data, these last two differences did not reach significant levels.

Since targets in the s_ITI condition appear more frequently than in the 1_ITI condition, it could be argued that the lower latencies of rats from s_ITI could be due to the more permanent orientation that these rats keep toward the panel. However, the presentation of the neutral cue previous to the target makes it possible for rats from both groups to orient toward the panel just before discrimination. This fact, along with 
the higher percentage of omissions and lower response-period rate observed in rats from group s_ITI than those from group 1_ITI, makes improbable an explanation based on permanent orientation for the differences in latency.

It has been suggested that high event rates (such as for s_ITI) in attentional tasks facilitates the occurrence of vigilance decrement in time (McGaughy and Sarter, 1995; Parasuraman, 1979; Parasuraman and See, 2000). Although measurements in different blocks of trials along time were not made, this could explain the higher percentage of omissions in rats from group s_ITI in comparison with rats from group 1_ITI.

In a parametric study of cued target detection in rats, Bushnell (1995) found that presentation of a neutral cue instead of no cue on $30 \%$ of the trials had little effect on either accuracy or latency. In our study, we found that the neutral cue is relevant for the rats to perform the visuospatial discrimination, especially under conditions of a prolonged and randomized ITI. There are several factors that can account for the differences between Bushnell's results and data from this study: use of a double cue instead of one central cue; presence of the neutral cue only on $30 \%$ of the trials; use of a five-second ITI; and finally, use of different stimulus onset asynchronies (i.e., time between the neutral cue and the target).

In contrast with several other models (e.g., Sahgal, 1988; Stoher, 1997), nonretractable levers were used in this study. The availability of the levers during the entire experiment rendered information about the variations in the rate of lever pressing during the different periods of the task, before and after removal of the neutral cue, as well as in the two groups with different ITI durations.

In relation to the convenience of using non-retractable levers, Bunsey and Strupp (1995), using an $\alpha 2$-adrenergic antagonist on a three-choice task with three different conditions (discrimination, vigilance, selective attention - distraction), observed that the injection of the drug inhibited premature responses (during the ITI) when presented with salient distractors. Thus, the availability of the levers during the entire task allowed them to obtain data about premature responses and, with that, a measure of impulsiveness (see also Eveden, 1999). Another reason against using retractable levers is their disruptive effect in the performance of the animals (Bushnell, 1998a; 1998b).

Our results constitute clear evidence of the relevance of the neutral cue in signaling the presentation of one of two stimuli for discrimination, thus dividing the task in a period of high expectancy and attentional load and a period of low expectancy and reallocation of attentional resources. Furthermore, they showed that this neutral cue is more critical for delimiting attentional load when the target is presented at a lower rate (1_ITI condition) and, thus, in a less predictable manner.

\section{ACKNOWLEDGEMENTS}

This work was supported by FONDECYT 1020970; CONICYT; PG-82-2003.

The authors would like to thank María de los Ángeles Saavedra, Fabián Soto, and Bethany Schneider for valuable comments on previous versions of the manuscript and to Fernando Vergara for technical assistance.

\section{REFERENCES}

BUNSEY MD, STRUPP BJ (1995) Specific effects of idazoxan in a distraction task: Evidence that endogenous norepinephrine plays a role in selective attention in rats. Behav Neurosci 109: 903-911

BUSHNELL PJ (1995) Overt orienting in the rat: Parametric studies of cued detection of visual targets. Behav Neurosci 109: 1095-1105

BUSHNELL PJ (1998a) Behavioral approaches to the assessment of attention in animals. Psychopharmacol 138: $231-259$

BUSHNELL PJ (1998b) Sustained attention and vigilance: A reply to Sarter and McGaughy. Psychopharmacol 138: 263-265

BUSHNELL PJ, KELLY KL, CROFTON KM (1994) Effects of toluene inhalation on detection of auditory signals in rats. Neurotoxicol Teratol 16: 149-160

CARLI M, ROBBINS TW, EVENDEN JL, EVERITT BJ (1983) Effects of lesions to ascending noradrenergic neurons on performance of a 5-choice serial reaction time task in rats: Implications for theories of dorsal noradrenergic bundle function based on selective attention and arousal. Behav Brain Res 9: 361-380 
DELANO PH, HAMAME CM, ROBLES L (2003) Delimited high expectancy period in a two choice task: Behavioral validation in the rat. Poster session presented in the Sixth IBRO world congress of neuroscience, Prague, Czech Republic

D'MELLO GD, STECKLER T (1996) Animal models in cognitive behavioural pharmacology: An overview. Cog Brain Res 3: 345-352

EVEDEN J (1999) Varieties of impulsivity. Psychopharmacol 146: 348-361

GIVENS B (1997) Effect of ethanol on sustained attention in rats. Psychopharmacol 129: 135-140

GRILLY DM, GOWANS GC (1988) Effects of naltrexone, and $d$-amphetamine, and their interaction on the stimulus control of choice behavior of rats. Psychopharmacol 96: 73-80

GRILLY DM, PISTELL PJ, SIMON BB (1998) Facilitation of stimulus detection performance of rats with $d$ amphetamine: a function of dose and level of training. Psychopharmacol 140: 272-278

MCGAUGHY J, KAISER T, SARTER M (1996) Behavioral vigilance following infusions of $192 \mathrm{IgG}-$ Saporin into the basal forebrain: Selectivity of the behavioral impairment and relation to cortical AChEPositive fiber density. Behav Neurosci 110: 247-265

MCGAUGHY J SARTER M (1995) Behavioral vigilance in rats: Task validation and effects of age, amphetamine, and benzodiazepine receptor ligands. Psychopharmacol 117: 340-357

MIRZA N, STOLERMAN I (1998) Nicotine enhances sustained attention in the rat under specific task conditions. Psychopharmacol 138: 266-274

MUIR JL (1996) Attention and stimulus processing in the rat. Cog Brain Res 3: 215-225
PANG K, MERKEL F, OLTON DS (1992) Expectancy and stimulus frequency: A comparative analysis in rats and humans. Percept Psychophys 51: 607-615

PANG K, WILLIAMS MJ, EGETH H, OLTON DS (1993) Nucleus basalis magnocellularis and attention: Effects of muscimol infusions. Behav Neurosci 107: 1031-1038

PARASURAMAN R (1979) Memory load and event rate control sensitivity decrements in sustained attention. Science 205: 924-927

PARASURAMAN R, SEE J (2000) Brain systems of vigilance. In: PARASURAMAN R (ed) The attentive brain. London: The MIT Press. pp: 189-220

POSNER MI (1980) Orienting of attention. Q J of Exp Psychol 32: 3-25

ROBBINS TW (2000) Arousal and attention: Psychopharmacological and neuropsychological studies in experimental animals. In: PARASURAMAN R (ed) The attentive brain. London: The MIT Press. pp: 189220

SAHGAL A (1988) Vasopressing and amphetamine, but not desglycinamide vasopressing, impair positively reinforced visual attention performance in rats. Behav Brain Res 29: 35-42

SARTER M, MCGAUGHY J (1998) Assessment of sustained and divided attention in rats: Aspects of validity. Psychopharmacol 138: 260-262

STECKLER T, MUIR JL (1996) Measurement of cognitive function: Relating rodent performance with human minds. Cog Brain Res 3: 299-308

STOHER JD, MOBLEY SL, ROICE D, BROOKS R, BAKER L, WILEY RG, ET AL. (1997) The effects of selective cholinergic basal forebrain lesions and aging upon expectancy in the rat. Behav Neurosci 76: 214227 